PHYSICAL REVIEW B 71, 239901(E) (2005)

\title{
Publisher's Note: Two-component density functional theory calculations of positron lifetimes for small vacancy clusters in silicon [Phys. Rev. B 71, 205215 (2005)]
}

\author{
D. V. Makhov and Laurent J. Lewis
}

(Received 7 June 2004; published 17 June 2005)

DOI: 10.1103/PhysRevB.71.239901

PACS number(s): 71.60.+z, 78.70.Bj, 71.55.Cn, 61.72.Bb, 99.10.Fg

This paper was published online on 31 May 2005 with a typographical error on page 2. The first sentence of the last paragraph in the right-hand column should read "The calculations were performed using the Vienna Ab-initio Simulation Package (VASP), which employs ...”. The paper has been corrected as of 7 June 2005. The text is correct in the printed version of the journal. 